EPJ Web of Conferences 41, 03021 (2013)

DOI: $10.1051 /$ epjconf/20134103021

(C) Owned by the authors, published by EDP Sciences, 2013

\title{
Ultrafast charge relocation in an ionic crystal probed by femtosecond $\mathrm{x}$-ray powder diffraction
}

\author{
M. Woerner ${ }^{\mathrm{a}}$, F. Zamponi, P. Rothhardt, J. Stingl, and T. Elsaesser \\ Max-Born-Institut für Nichtlineare Optik und Kurzzeitspektroskopie, 12489 Berlin, Germany
}

\begin{abstract}
Transient electron density maps of potassium dihydrogen phosphate $\left(\mathrm{KH}_{2} \mathrm{PO}_{4}\right.$, KDP) are derived from femtosecond $\mathrm{x}$-ray powder diffraction patterns. Upon photoexcitation, the low-frequency TO soft mode is elongated impulsively and modulates the electronic charge distribution on the length scale of interatomic distances, much larger than the vibrational amplitude of the nuclear motion. The results demonstrate a charge transfer from the volumes around the $\mathrm{P}$-atoms to those containing the $\mathrm{O}-\mathrm{H} \cdots \mathrm{O}$ units and a quadrupolar distortion of the $\mathrm{K}^{+}$charge distribution. This behavior reflects the interplay of nuclear motions and electric polarizations in the ionic crystal lattice.
\end{abstract}

Ionic crystals are characterized by a pronounced coupling of electronic and lattice degrees of freedom. Elongations of lattice modes, in particular of the so-called soft-mode phonon, result in a relocation of electronic charge and play an important role for transitions between different structural phases, e.g., from a ferroelectric to a paraelectric lattice geometry. Such phenomena have been analyzed by structure research under quasi-stationary conditions and by theoretical calculations. However, the coupled dynamics of lattice motions and charge relocations which is expected to occur on ultrafast time scales has remained mainly unaddressed. Insight into such nonequilibrium processes requires structure probes which combine femtosecond temporal and (sub-)atomic spatial resolution.

Here, we apply x-ray powder diffraction with a time resolution of 100 fs $[1,2]$ to study the coupled lattice and charge dynamics in the prototype material potassium dihydrogen phosphate $\mathrm{KH}_{2} \mathrm{PO}_{4}$ (KDP). Highly accurate measurements of transient diffraction patterns consisting of many DebyeScherrer rings allow for a reconstruction of transient maps of electronic charge densities, in this way providing direct access to the transient charge distribution in the material. We observe ultrafast strong charge relocations on the length scale of a chemical bond which are induced by coherent soft-mode elongations of much smaller spatial amplitude.

In the paraelectric phase studied here, KDP has a tetragonal structure with $I \overline{4} 2 d$ symmetry [3]. The unit cell contains $4 \mathrm{KH}_{2} \mathrm{PO}_{4}$ units and has dimensions of $\mathrm{a}=\mathrm{b}=0.74529 \mathrm{~nm}$ and $\mathrm{c}=0.69751 \mathrm{~nm}$ at $\mathrm{T}=298 \mathrm{~K}$ (Fig.1a). The light-blue plane in Fig. 1a is parallel to the Z-axis, goes through the upper $\mathrm{K}$ atom, the central $\mathrm{P}$ atom and minimizes the overlap with the $\mathrm{O}$ atoms of the $\mathrm{PO}_{4}$ tetrahedron. It is slightly shifted along $\mathrm{c}$ in order to completely include the $\mathrm{K}$ atom. Fig. 1b shows the stationary distribution of electronic charge in this plane as derived from the known equilibrium structure and the atomic form factors. For an analysis of transient charge density maps, the unit cell was divided in sub-volumes around the different atoms which are indicated as solid lines.

The $\mathrm{x}$-ray diffraction experiment was performed in an optical-pump - x-ray-probe scheme. The sample was excited by sub-50 fs pulses at $266 \mathrm{~nm}$, exploiting the two-photon interband absorption $\left(\mathrm{E}_{\text {gap }} \approx 7-8 \mathrm{eV}\right)$. The resulting transient structural changes were mapped by diffracting 100-fs hard $\mathrm{x}-$ ray pulses from the excited sample. The x-ray pulses were generated in a laser-driven plasma source, providing $\mathrm{Cu}-\mathrm{K} \alpha$ pulses at a wavelength of $\lambda_{\mathrm{x}-\text { ray }}=0.154 \mathrm{~nm}$. Diffraction patterns consisting of up to 20 Debye-Scherrer rings were collected by a large-area CCD detector. A typical pattern together

\footnotetext{
a e-mail: woerner@mbi-berlin.de
}

This is an Open Access article distributed under the terms of the Creative Commons Attribution License 2.0, which permits unrestricted use, distribution, and reproduction in any medium, provided the original work is properly cited. 
(a)
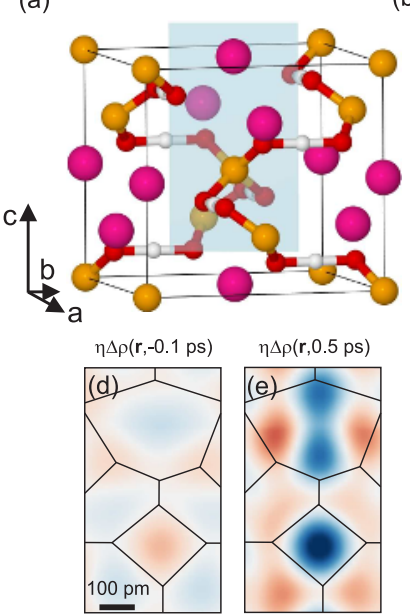

$-43 \mathrm{e}^{-} / \mathrm{nm}^{3}$

(h)

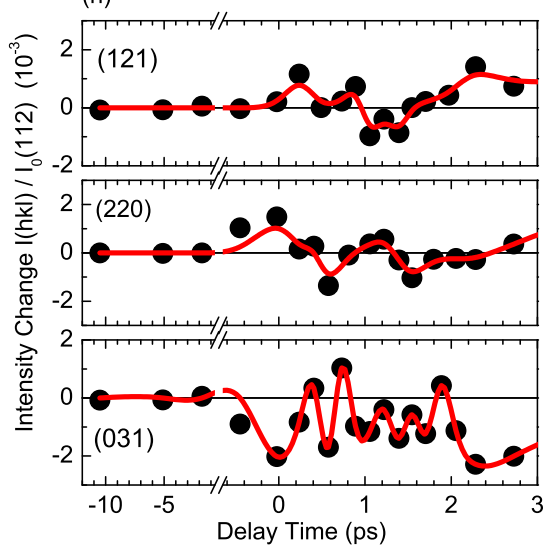

(b)
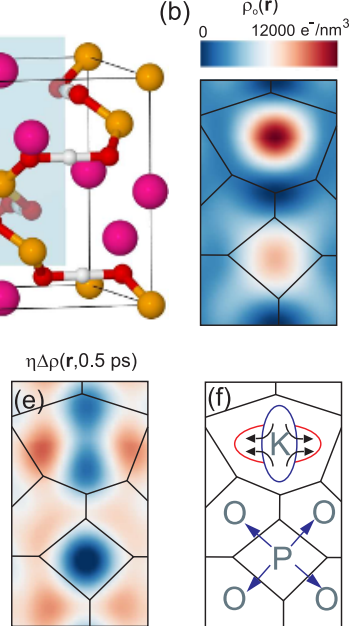

$43 \mathrm{e}^{-} / \mathrm{nm}^{3}$ $m^{3}$$$
\text { (1) }
$$

(c)
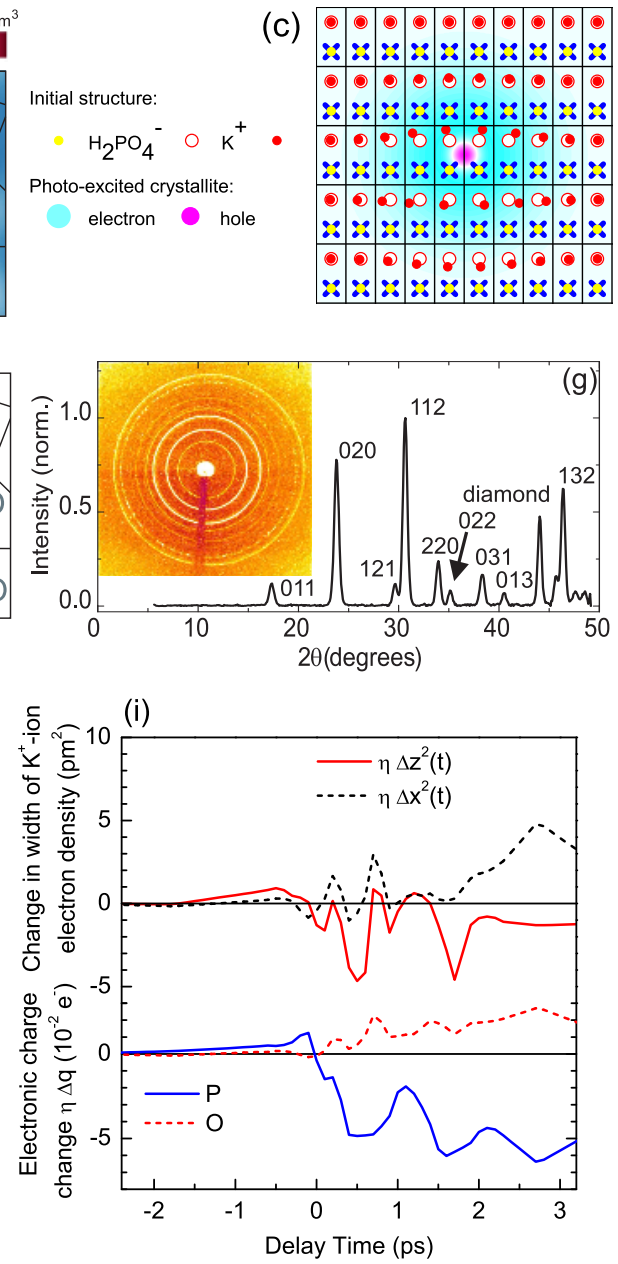

Fig. 1. (a) Unit cell of KDP in the paraelectric phase (yellow spheres: phosphorous atoms (P), pink: potassium $(\mathrm{K})$, red: oxygen $(\mathrm{O})$, white: hydrogen $(\mathrm{H})$ ). (b) Equilibrium electron density map $\rho_{0}(\mathbf{r})$ in the plane defined by the rectangle in (a). The solid lines represent the system of polyhedrons enclosing the different atoms used in data analysis. (c) Schematic of a Frenkel exciton which is generated by the optical pump pulse and extends over many unit cells. (d,e) Differential charge density maps for pump-probe delays of -0.1 and +0.5 ps as derived from the diffraction data. (f) Positions of atoms in the plane together with a schematic view of the main features emerging from the measurements: charge transfer from $\mathrm{P}$ atom to the $\mathrm{O}$ atoms and the prolate-oblate deformation of the charge around the $\mathrm{K}$ atom. (g) Typical powder pattern acquired after 7 min exposure time. Inset: raw data from the CCD detector with the ring pattern. (h) Change of diffracted intensity on three selected reflections as a function of the delay between optical pump pulse and the x-ray probe. (i) Upper panel: Transient quadrupolar distortion of the electron density of the $\mathrm{K}^{+}$ion: the broadening along the c-axis $\eta \Delta z^{2}(t)$ and along the a/b axis $\eta \Delta x^{2}(t)$ are plotted vs. pump-probe delay. Lower panel: Time-resolved charge changes $\eta \Delta q(t)$ on the $\mathrm{P}$ and $\mathrm{O}$ atoms.

with the raw data as acquired by the detector is shown in Fig. 1g. To reconstruct transient changes of electronic charge density, i.e., determine differential charge density maps in the ultrafast time domain, we extended a method for reconstructing the electron density in centrosymmetric materials [1] to the non-centrosymmetric KDP [4].

In Fig. 1h, the change of diffracted intensity is plotted as a function of pump-probe delay for 3 different reflections of the pattern shown in Fig. 1g. Intensity changes as small as $2 \times 10^{-3}$ are detected and display an oscillatory time evolution. From the complete data set, we derived the transient charge 
density maps of Fig. 1d,e, where the electron density $\eta \Delta \rho(\mathbf{r}, t)=\eta\left[\rho_{e x}(\mathbf{r}, t)-\rho_{0}(\mathbf{r},-\infty)\right]$ in the plane indicated in Fig. 1a is plotted for two pump-probe delays. Here, $\eta \ll 1$ is the ratio between excited and unexcited unit cells. The main features emerging from the measurements are a charge transfer from the $\mathrm{P}$ atom to the surrounding $\mathrm{O}$ atoms and an oblate-prolate deformation of the electron distribution around the $\mathrm{K}$ atom, as shown schematically in Fig. 1f. For a quantitative analysis, the total charge and the quadrupolar distortion of the corresponding electronic density distribution in the polyhedrons were derived (Fig.1i). In the upper panel, the prolate-to-oblate transition around time delay zero and the subsequent oblate oscillations are shown. The data in the lower panel demonstrate a charge transfer from the $\mathrm{P}$ atom to the surrounding $\mathrm{O}$ atoms. Fourier analysis of the oscillatory components gives frequencies in agreement with those of the low-frequency LO and TO phonons of KDP, the latter representing the soft mode.

The physical mechanisms of the observed charge relocations are illustrated schematically in Fig. 1c: two-photon excitation of KDP creates a localized Frenkel exciton and induces a strong change of the electric polarizability ( $E_{\text {exciton }} \approx 1 \mathrm{eV}$, Bohr radius $a_{B} \approx 1 \mathrm{~nm}$ ). The strong displacement field within the exciton Bohr radius impulsively launches coherent wavepacket motions of low-frequency polar phonons, among them the TO soft mode. The soft mode oscillations induce the large-amplitude charge relocations, due to the altered electronic polarizability in the excited state. Such dynamics is in line with the Cochran theory of ferroelectricity [5].

\section{References}

1. M. Woerner, F. Zamponi, Z. Ansari, J. Dreyer, B. Freyer, M. Prémont-Schwarz and T. Elsaesser, J. Chem. Phys. 133, 064509 (2010).

2. F. Zamponi, Z. Ansari, M. Woerner and T. Elsaesser, Opt. Express 18, 947 (2010).

3. R. J. Nelmes, Z. Tun and W. F. Kuhs, Ferroelectrics 71, 125 (1987).

4. F. Zamponi, P. Rothhardt, J. Stingl, M. Woerner and T. Elsaesser, Proc. Nat. Acad. Sci. USA, 109, 5207 (2012).

5. W Cochran, Adv. Phys. 9, 387 (1960). 